\title{
Simulation of environmental impact of an existing natural gas dehydration plant using a combination of thermodynamic models
}

\author{
Mehdi Amouei Torkmahalleh, Galiya Magazova, Aliya Magazova, Seyed Jamal Hassani \\ Rad
}

Department of Chemical Engineering

\section{Abstract}

A new approach was presented to improve the simulation results of an existing TEG based natural gas dehydration plant, using Aspen Plus software. Furthermore, the environmental impact of the plant was investigated. The plant consists of four main unit operations including an absorber, a flash tank, a stripper and a regenerator. Twelve thermodynamic models were assigned to these units. In the first step of the study, only one thermodynamic model was assigned to all of the units while in other steps, combinations of thermodynamic models were employed. The most accurate model combination was found to be RKSMHV2 for the absorber and stripper and PSRK for the flash tank and regenerator. It was found that a proper combination of thermodynamic models may improve the simulation results. As solvent circulation rate increased, BTEX, VOC and greenhouse gas emissions enhanced.

Original language

Pages (from-to)

Number of pages

Journal

Volume

State

\section{English}

$38-47$

10

Process Safety and Environmental Protection

104

Published - Nov 12016

Amouei Torkmahalleh, M., Magazova, G., Magazova, A., \& Hassani Rad, S. J. (2016). Simulation of environmental impact of an existing natural gas dehydration plant using a combination of thermodynamic models. Process Safety and Environmental Protection, 104, 3847. DOI: 10.1016/j.psep.2016.08.008 